\title{
Cuidados de enfermagem para o manejo adequado de náuseas e vômitos em mulheres com câncer de mama em terapia antineoplásica parenteral: revisão integrativa
}

\author{
Nursing care for the proper management of nausea and vomiting in women with breast \\ cancer undergoing parenteral antineoplastic therapy: integrative review
}

Cuidados de enfermería para el manejo adecuado de náuseas y vómitos en mujeres con cáncer de mama en terapia antineoplásica parenteral: revisión integrativa

Valéria da Costa Gomes ${ }^{1 *}$, Ana Flávia de Paula Rodrigues Reginaldo ${ }^{1}$, Orácio Carvalho Ribeiro Junior $^{1}$, Genice Lemos Campos ${ }^{1}$, Jéssica Karoline Alves Portugal ${ }^{2}$, Marcelo Henrique da Silva Reis ${ }^{2}$, José Carlos Ferreira Pinheiro Junior², Sibele Naiara Ferreira Germano², Yara da Silva dos Reis², Sara Moriá Rodrigues Barbosa².

\section{RESUMO}

Objetivo: Identificar na literatura as condutas de enfermagem não farmacológicas frente ao manejo adequado de náuseas e vômitos de acordo com o potencial emetogênico dos protocolos de Terapia Antineoplásica em mulheres com câncer de mama. Métodos: Trata-se de uma revisão Integrativa da Literatura com busca por artigos científicos na Base de Dados em Enfermagem (BDENF), Literatura Latino Americana e do Caribe em Ciências da Saúde (LILACS), Sistema Online de Busca e Análise de Literatura Médica (MEDLINE), National Library of Medicine (PUBMED) e Scientific Eletronic Library Online (SCIELO). Resultados: Foram identificados 104 artigos, após o processo de busca e análise, restaram 15, que foram incluídos como amostra final deste estudo. Foi realizado o cálculo do (PE) para definir a classificação dos protocolos em: Alto (>90\% de chances de náuseas e vômitos durante a TA), Moderado (de 30-90\%) e Baixo (de 10-30\%). No total, foram contabilizados 48 protocolos estabelecidos para realização da terapia antineoplásica, destes, $29,2 \%(\mathrm{~N}=14)$ Alto, 39,6\% ( $\mathrm{N}=19)$ Moderado, 22,9\% ( $\mathrm{N}=11)$ Baixo e 8,3\%(N=4) Mínimo. Considerações finais: $O$ estudo apontou que as principais condutas estão baseadas na implementação de atividades educativas que promovam o conhecimento adequado da paciente em relação ao tratamento e seus possíveis efeitos colaterais.

Palavras chave: Enfermagem, Câncer de mama, Quimioterapia.

\section{ABSTRACT}

Objective: To identify non-pharmacological nursing practices in the literature regarding adequate management of nausea and vomiting according to the emetogenic potential of the protocols for Antineoplastic Therapy in women with breast cancer. Methods: This is an Integrative Literature review with search for scientific articles in the Nursing Database (BDENF), Latin American and Caribbean Literature in Health Sciences (LILACS), Online Medical Literature Search and Analysis System (MEDLINE), National Library of Medicine (PUBMED) and Scientific Electronic Library Online (SCIELO). Results: 104 articles were identified, after the search and analysis process, 15 remained, which were included as the final sample of this study. Calculation of (PE) was performed to define the classification of the protocols in: High ( $>90 \%$ chances of nausea and vomiting during ED), Moderate (30-90\%) and Low (10-30\%). In total, 48 protocols established for carrying out antineoplastic therapy were accounted for, of these, $29.2 \%(\mathrm{~N}=14)$ High, $39.6 \%(\mathrm{~N}=19)$ Moderate, 22.9\% ( $N=11)$ Low and $8,3 \%(N=4)$ Minimum. Final considerations: The study pointed out that the main conducts are based on the implementation of educational activities that promote adequate knowledge of the patient in relation to the treatment and its possible side effects.

Key words: Nursing, Breast neoplasms, Chemotherapy.

1Universidade do Norte (UNINORTE), Manaus - AM. *E-mail: valeriaenfa26@outlook.com

2Universidade Federal do Amazonas (UFAM), Coari - AM.

SUBMETIDO EM: 3/2020

ACEITO EM: 4/2020

PUBLICADO EM: 8/2020 


\section{RESUMEN}

Objetivo: identificar las prácticas de enfermería no farmacológicas en la literatura sobre el manejo adecuado de las náuseas y los vómitos de acuerdo con el potencial emetógeno de los protocolos de terapia antineoplásica en mujeres con cáncer de seno. Métodos: Esta es una revisión de Literatura Integrativa con búsqueda de artículos científicos en la Base de Datos de Enfermería (BDENF), Literatura Latinoamericana y del Caribe en Ciencias de la Salud (LILACS), Sistema de Búsqueda y Análisis de Literatura Médica en línea (MEDLINE), Biblioteca Nacional de Medicina (PUBMED) y Scientific Electronic Library Online (SCIELO). Resultados: Se identificaron 104 artículos, después del proceso de búsqueda y análisis, quedaron 15, que se incluyeron como la muestra final de este estudio. El cálculo de (PE) se realizó para definir la clasificación de los protocolos en: Alta (> 90\% de posibilidades de náuseas y vómitos durante la DE), Moderada (30-90\%) y Baja (10-30\%). En total, se contabilizaron 48 protocolos establecidos para llevar a cabo la terapia antineoplásica, de estos, 29.2\% ( $N=14)$ Alto, 39.6\% ( $N=19)$ Moderado, 22.9\% ( $N=11)$ Bajo y 8,3\% $(N=4)$ Mínimo. Consideraciones finales: El estudio señaló que las principales conductas se basan en la implementación de actividades educativas que promueven el conocimiento adecuado del paciente en relación con el tratamiento y sus posibles efectos secundarios.

Palabras clave: Enfermería, Neoplasias de mama, Quimioterapia.

\section{INTRODUÇÃO}

O câncer de mama é uma doença devastadora, gerado por células malignas desordenadas que formam um tumor geneticamente modificado, possuindo células que se dividem rapidamente, tornando-se agressivas e com o potencial de invadir órgãos e tecidos. O principal tipo histológico de câncer que apresenta maior proporção entre o sexo feminino é o carcinoma ductal infiltrante, originado nos ductos mamários, representando cerca de $80 \%$ a $90 \%$ dos casos (INCA, 2018).

A Organização Mundial de Saúde (OMS) aponta que o câncer de mama é o segundo mais frequente no mundo, com estimativa de 2,09 milhões de novos casos em 2018 (WHO, 2018). Em âmbito nacional, foram estimados 59.700 casos novos para o biênio 2018-2019, considerando 56,33 casos a cada 100 mil mulheres (INCA, 2017).

Neste contexto, o Ministério da Saúde recomenda novas diretrizes que auxilie no rastreamento do câncer de mama: a mamografia, ultrassonografia, ressonância magnética, termografia e tomossíntese, além do autoexame e exame clínico das mamas, no entanto, todos desempenham papel central na detecção, no diagnóstico e na conduta das doenças mamárias (MIGOWSKI A, et al., 2018).

Para realizar o tratamento da neoplasia são utilizados basicamente as seguintes modalidades: cirúrgica, radioterapia, terapia hormonal, imunoterapia e Terapia Antineoplásica (TA), conhecida como quimioterapia, sendo utilizada em combinação ou isoladamente (CONCEIÇÃO LL, et al., 2008). O câncer de mama avançado geralmente é tratado com quimioterapia adjuvante, sendo realizada antes do procedimento cirúrgico ou neoadjuvante, após o procedimento cirúrgico (BRASIL, 2018).

Neste sentido, compreende-se que o tratamento é eficaz, pois contribui na erradicação de micrometástase e diminui a chance de recidiva tumoral. Porém, a quimioterapia pode causar eventos adversos severos como: alopecia, mucosite, neutropenia, náuseas, vômitos, dentre outros (FRAZÃO A e SKABA MMF, 2013).

O principal efeito colateral da TA são náuseas e vômitos. A Náusea é caracterizada como sensação pertinente a um desconforto gástrico tendencioso a expressa vontade de vomitar, podendo também estar associada à salivação, palidez, bradicardia ou taquicardia e/ou midríase. O vômito ou êmese é conceituado como eliminação involuntária do conteúdo gástrico através da boca (SÁ CU, et al., 2014; FERNANDES DT, et al., 2018).

O potencial emetogênico das drogas é caracterizado pelo nível de gravidade desses eventos adversos, podendo ser alto (> que $90 \%$ ), como exemplos temos a ciclofosfamida (>1500 mg/m2) e cisplatina, moderado (em torno de 30 a $90 \%$ ) ciclofasfamida ( $<1500 \mathrm{mg} / \mathrm{m} 2)$, epirrubicina e doxorrubucina, baixo (de 10 a $30 \%) 5-$ fluouracil, paclitaxel, docetaxel, e mínimo (<que10\%) vinorelbine (GOZZO TO, et al., 2013). 
De acordo com este potencial, as condutas de enfermagem podem ser diferenciadas para cada protocolo quimioterápico. Por outro lado, estudos apontam que na maioria dos casos, as pacientes recebem apenas orientações quanto ao manejo farmacológico que atua na prevenção e controle das náuseas e vômitos, deixando lacunas em relação às informações relevantes sobre medidas não farmacológicas (tipo de alimentação adequada, nutrição dietética fracionada, a importância da ingesta hídrica) e medidas educativas (MOLASSIOTIS A, 2008; GOZZO TO, et al., 2014; FERNANDES DT, et al., 2018).

Através da escuta inicial dos usuários com câncer, o enfermeiro e demais profissionais de saúde têm a possibilidade de manter um relacionamento interpessoal com os pacientes e familiares, aconselhando-os sobre a enfermidade, suas consequências, desafios e tratamento, cujo intuito é diminuir o impacto que esse processo doloroso trará. Esta etapa é fundamental, pois vai dar oportunidade de cada um participar da discussão das melhores estratégias para o enfrentamento da doença (BRASIL, 2008).

Diante deste panorama, cabe ao profissional proporcionar de forma integral uma assistência qualificada, antes, durante e depois do tratamento antineoplásico por meio da educação em saúde que assegure um plano terapêutico adequado e que possibilite a paciente melhor adesão ao tratamento.

A partir dessas informações questiona-se: quais as condutas de enfermagem para o manejo adequado de náuseas e vômitos em mulheres com câncer de mama que realizam a Terapia Antineoplásica?

Desta forma, o presente estudo tem como objetivo identificar através de uma revisão integrativa da literatura as condutas de enfermagem não farmacológicas frente a náuseas e vômitos de acordo com o potencial emetogênico dos protocolos de Terapia Antineoplásica em mulheres com câncer de mama.

\section{MÉTODOS}

Trata-se de uma revisão integrativa de literatura, além disto, este estudo foi metodologicamente dividido em seis etapas, para permitir que seja construído de maneira minuciosa e sistematizada.

Primeira Etapa: Trata-se da formulação da questão norteadora, que guiou o estudo acerca do tema proposto, formulado a partir da seguinte instigação: quais as condutas de enfermagem para o manejo adequado de náuseas e vômitos em mulheres com câncer de mama que realizam a Terapia Antineoplásica?

A partir disto, foi esclarecido a hipótese, identificado o tema e definidos os objetivos deste estudo. Posteriormente, foi realizada a definição dos descritores a serem utilizados e a partir de então, foi dado início a produção do estudo em questão.

Diante disto, foi realizado um levantamento bibliográfico, por estudos publicados em forma de artigos, contidos na Biblioteca Virtual em Saúde (BVS), indexados nas bases de dados Base de Dados em Enfermagem (BDENF), Literatura Latino Americana e do Caribe em Ciências da Saúde (LILACS), Sistema Online de Busca e Análise de Literatura Médica (MEDLINE), National Library of Medicine (PUBMED) e Scientific Eletronic Library Online (SCIELO), utilizando os descritores em português "câncer de mama", "náuseas", "vômito", "quimioterapia" e "cuidados de enfermagem", segundo definidos pelos Descritores em Ciência em Saúde (DeCs), separada pelo operador boleano AND e descritores inglês "breast cancer", "nauseas", "vomit", "chemotherapy" e "nursing care".

Segunda Etapa: Nesta fase, estabeleceu-se os critérios de inclusão e exclusão dos artigos, para obter o quantitativo final de publicações a serem utilizadas na revisão integrativa da literatura. Foram incluídos, artigos originais e com o texto completo disponível, publicados no período de janeiro de 2008 a junho de 2018, artigos no idioma português e inglês com a temática relevante ao estudo.

Foram excluídos os artigos que não tinham acesso gratuito, relatos de experiência, estudos de caso e estudos da literatura cinza. Estudos repetidos em mais de uma base de dados, foram considerados apenas em sua primeira aparição nos resultados.

Terceira Etapa: Foi realizada a coleta de dados e caracterização dos dados obtidos em um instrumento elaborado pelos pesquisadores, que inclui informações relevantes para a sistematização do estudo, dados de identificação do artigo e periódico, suas características metodológicas e seus objetivos (Base de dados, 
Revista, Título, Autor (es), Objetivo, Metodologia e Ano). Esta caracterização dos artigos permite a classificação e identificação de maneira mais ágil para o pesquisador, evitando informações desnecessárias e a releitura dos estudos.

Quarta Etapa: Nesta etapa, foi realizada a análise dos estudos obtidos, conforme a leitura das publicações, pôde-se observar diferentes perspectivas sobre o tema, envolvendo experiências específicas que serviram como um pilar para a construção deste estudo.

Quinta Etapa: Para a discussão dos resultados obtidos durante a análise dos estudos, foi necessário apresentar as mais diferentes abordagens expostas pelos autores, para assim, construir uma síntese de conteúdos realmente relevantes para o estudo.

Sexta Etapa: A apresentação dos dados obtidos foi feita de maneira descritiva e quantitativa. Ainda na seção de resultados, compilou-se um gráfico com o cálculo do potencial emetogênico das drogas segundo as Diretrizes Internacionais do National Comprehensive Cancer Network (NCCN).

\section{RESULTADOS}

Nesta pesquisa foram identificados 104 artigos: 22 do Banco de Dados em Enfermagem (BDENF), 12 Literatura Latino-americana do Caribe em Ciências da Saúde (LILACS), 8 do Sistema Online de Busca e Análise de Literatura Médica (MEDLINE), 37 da Scientific Electronic Library Online (ScIELO) e 25 do National Library of Medicine (PUBMED).

Após o processo de busca, análise primária dos títulos, resumos e aplicação dos critérios de inclusão e exclusão, restaram 15 artigos, equivalentes a $14,4 \%$ de todos os artigos identificados no processo de busca, os 15 foram incluídos como amostra final deste estudo, conforme evidenciando na Tabela 1.

Tabela 1 - Metodologia de busca e seleção dos estudos incluídos na revisão.

\begin{tabular}{lcc}
\multirow{2}{*}{ Base de dados acessada } & \multicolumn{2}{c}{ Publicações incluídas } \\
\cline { 2 - 3 } & $\mathbf{N}$ & $\%$ \\
\hline BDENF & 2 & 13,3 \\
\hline LILACS & 1 & 6,7 \\
\hline MEDLINE & 1 & 6,7 \\
\hline PUBMED & 5 & 33,3 \\
\hline ScIELO & 6 & 40,0 \\
\hline Total & 15 & 100
\end{tabular}

N= Número de artigos ao final do processo de busca e análise.

Fonte: Gomes VC, et al., 2019.

Em uma análise geral dos estudos, nota-se uma prevalência maior de publicações na base de dados ScIELO, seguido pelas bases PUBMED, BDENF, LILACS e MEDLINE. Observa-se também que a maioria das publicações estão em revistas brasileiras de saúde em enfermagem, mostrando o crescimento de publicações na última década e a amplitude de conhecimento relacionado a temática abordada. A revisão mostra que a maior parte dos estudos estão no escopo metodológico descritivo exploratório de abordagem quantitativa, segundo as descrições do Quadro 1.

Para a construção do gráfico dos potenciais emetogênicos (PE), primeiramente foi realizada a separação dos principais guidelines nacionais e internacionais, considerados a referência mundial para escolha dos protocolos de terapia antineoplásica (TA), oriundos dos seguintes países: Brasil, Canadá, Estados Unidos e Áustria. Os protocolos foram avaliados individualmente segundo o nome e dose padrão da droga. 
Na sequência, foi realizado o cálculo do (PE) de cada protocolo de TA para definir a classificação dos protocolos em: PE Alto (maior que $90 \%$ de chances de náuseas e vômitos durante a TA), PE Moderado (de 30-90\%) e PE Baixo (de 10-30\%). No total, foram contabilizados 48 protocolos estabelecidos para realização da terapia antineoplásica, destes, 29,2\% ( $N=14)$ de PE Alto, 39,6\% ( $N=19)$ PE Moderado, 22,9\% ( $N=11)$ de PE Baixo e 8,3\%(N=4) de PE Mínimo. Conforme demonstrado na Figura 1.

Figura 1 - Potencial emetogênico dos protocolos da TA para neoplasia maligna das mamas.

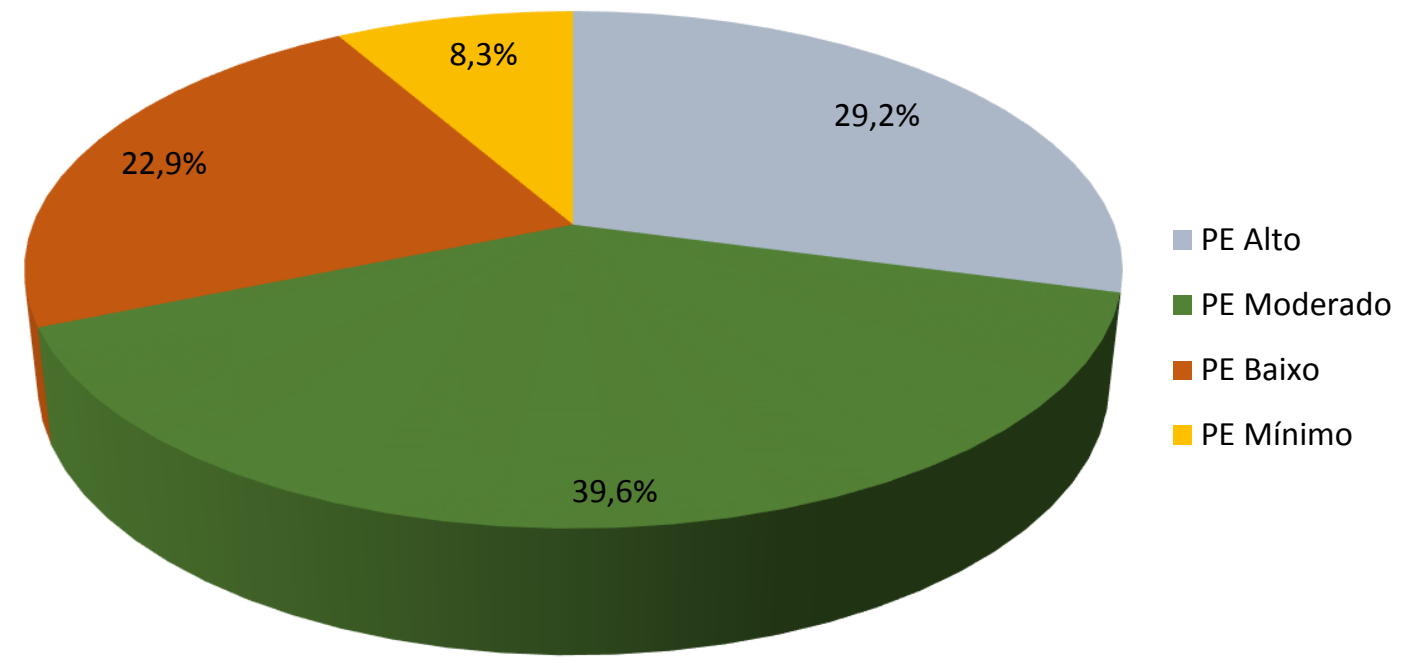

Fonte: Gomes VC, et al., 2019.

Em relação à categorização dos artigos, observou-se a enfermagem como a área onde o interesse pela temática foi mais preponderante, em seguida, nota-se uma prevalência menos acentuada da área de medicina. Quanto à formação dos autores, a sua maioria é apresentada por enfermeiros, em relação à titulação, a mais prevalente foi a de Doutor em enfermagem, seguidos por Mestres e Bacharéis. Conforme exposto no Quadro 2. 
Quadro 1 - Caracterização dos estudos a partir dos seguintes tópicos: Base, Revista, Título, Autor (es), Objetivo, Metodologia e Ano.

\begin{tabular}{|c|c|c|c|c|c|c|c|c|}
\hline \multirow[b]{2}{*}{ № } & \multirow[b]{2}{*}{ Base } & \multirow[b]{2}{*}{ Revista } & \multirow[b]{2}{*}{ Título } & \multirow[b]{2}{*}{ Autor (es) } & \multirow[b]{2}{*}{ Objetivo } & \multicolumn{2}{|c|}{ Metodologia } & \multirow[b]{2}{*}{ Ano } \\
\hline & & & & & & $\begin{array}{l}\text { Tipo de } \\
\text { estudo }\end{array}$ & Abordagem & \\
\hline 1 & BDENF & $\begin{array}{l}\text { Revista de } \\
\text { enfermagem } \\
\text { UFPE On Line }\end{array}$ & $\begin{array}{l}\text { O enfermeiro na prática educativa junto } \\
\text { aos familiares de clientes com câncer de } \\
\text { mama submetidas à quimioterapia }\end{array}$ & $\begin{array}{l}\text { Oliveira DFL } \\
\text { e Valente } \\
\text { GSC. }\end{array}$ & $\begin{array}{c}\text { Identificar como se dá o trabalho dos } \\
\text { enfermeiros no atendimento às mulheres } \\
\text { com câncer de mama em quimioterapia } \\
\text { antineoplásica. }\end{array}$ & Exploratório & Qualitativa & 2014 \\
\hline 2 & BDENF & $\begin{array}{l}\text { Revista de } \\
\text { enfermagem } \\
\text { UFPE On Line }\end{array}$ & $\begin{array}{l}\text { Implementação de manuais educativos } \\
\text { na consulta de enfermagem: opinião dos } \\
\text { pacientes submetidos à quimioterapia } \\
\text { antineoplásica }\end{array}$ & $\begin{array}{l}\text { Cruz FOAM, } \\
\text { et al. }\end{array}$ & $\begin{array}{l}\text { Conhecer a opinião dos pacientes com } \\
\text { relação aos manuais educativos } \\
\text { implementados durante a consulta de } \\
\text { Enfermagem prévia à quimioterapia } \\
\text { antineoplásica. }\end{array}$ & $\begin{array}{l}\text { Descritivo, } \\
\text { exploratório, } \\
\text { transversal e } \\
\text { prospectivo }\end{array}$ & Qualitativa & 2017 \\
\hline 3 & LILACS & $\begin{array}{l}\text { Revista de } \\
\text { Enfermagem } \\
\text { UERJ }\end{array}$ & $\begin{array}{l}\text { Mulheres com câncer de mama: ações } \\
\text { de autocuidado durante a quimioterapia }\end{array}$ & $\begin{array}{l}\text { Gonçalves } \\
\text { LLC, et al. }\end{array}$ & $\begin{array}{c}\text { Traçar um perfil das práticas de autocuidado } \\
\text { das pacientes com CA de mama de um } \\
\text { ambulatório público de oncologia em } \\
\text { Sergipe. }\end{array}$ & $\begin{array}{l}\text { Descritivo, } \\
\text { exploratório }\end{array}$ & Quantitativa & 2009 \\
\hline 4 & MEDLINE & $\begin{array}{l}\text { Online Journal } \\
\text { Brasillian of } \\
\text { Nursing - OBJN }\end{array}$ & $\begin{array}{l}\text { Qualidade de vida e pessoas em uso de } \\
\text { antineoplásicos: estudo descritivo }\end{array}$ & Sá CU, et al. & $\begin{array}{l}\text { Avaliar a qualidade de vida dos pacientes } \\
\text { em tratamento antineoplásico que } \\
\text { apresentaram náuseas e vômitos. }\end{array}$ & Descritivo & Quantitativa & 2014 \\
\hline 5 & PUBMED & $\begin{array}{c}\text { Journal of } \\
\text { alternative and } \\
\text { complementary } \\
\text { medicine }\end{array}$ & $\begin{array}{l}\text { Efeitos da massagem terapêutica na } \\
\text { qualidade de vida em pacientes com } \\
\text { câncer de mama durante o tratamento }\end{array}$ & $\begin{array}{l}\text { Sturgeon M, } \\
\quad \text { et al. }\end{array}$ & $\begin{array}{c}\text { Avaliar o impacto da massagem terapêutica } \\
\text { na qualidade de vida de pacientes em } \\
\text { tratamento para câncer de mama. }\end{array}$ & Prospectivo & Qualitativa & 2009 \\
\hline 6 & PUBMED & $\begin{array}{l}\text { Iranian Red } \\
\text { Crescent } \\
\text { Medical Journal }\end{array}$ & $\begin{array}{c}\text { Efeito da terapia de ervas para náuseas } \\
\text { e vômitos induzidos por quimioterapia } \\
\text { em pacientes com câncer }\end{array}$ & $\begin{array}{l}\text { Montazeri } \\
\text { SA, et al. }\end{array}$ & $\begin{array}{c}\text { Avaliar a eficácia do gengibre complementar } \\
\text { entre pacientes com câncer que } \\
\text { experimentam náuseas e vômitos. }\end{array}$ & Prospectivo & Quantitativa & 2013 \\
\hline 7 & PUBMED & $\begin{array}{l}\text { Support Care } \\
\text { Cancer }\end{array}$ & $\begin{array}{c}\text { Redução da anorexia, náusea e êmese } \\
\text { induzidas pela quimioterapia através de } \\
\text { uma intervenção estruturada de } \\
\text { enfermagem: um estudo multicêntrico } \\
\text { por grupo randomizado }\end{array}$ & $\begin{array}{l}\text { Jahn } P \text {, et } \\
\text { al. }\end{array}$ & $\begin{array}{c}\text { Avaliar o programa de Melhoria do } \\
\text { Autocuidado pela Enfermagem Oncológica } \\
\text { (SCION) para reduzir a anorexia, } \\
\text { a náusea e a emese angustiante (ANE) em } \\
\text { pacientes com câncer submetidos } \\
\text { à quimioterapia. }\end{array}$ & Comparativo & Quantitativa & 2009 \\
\hline 8 & PUBMED & $\begin{array}{l}\text { Clinical Journal } \\
\text { of Oncology } \\
\text { Nursing }\end{array}$ & $\begin{array}{l}\text { Ingestão oral de gengibre para náuseas } \\
\text { e vômitos induzidos por quimioterapia } \\
\text { entre mulheres com câncer de mama }\end{array}$ & $\begin{array}{l}\text { Arslan M e } \\
\text { Ozdemir L. }\end{array}$ & $\begin{array}{c}\text { Avaliar o efeito do gengibre } \\
\text { sobre náuseas e vômitos relacionados } \\
\text { à quimioterapia. }\end{array}$ & $\begin{array}{l}\text { Experimental } \\
\text { randomizado- } \\
\text { controlado }\end{array}$ & $\begin{array}{l}\text { Quali- } \\
\text { quantitativa }\end{array}$ & 2015 \\
\hline
\end{tabular}

REAS/EJCH | Vol.Sup.n.53 | e3517 | DOI: https://doi.org/10.25248/reas.e3517.2020 Página $\mathbf{6}$ de 13 


\section{Revista Eletrônica Acervo Saúde / Electronic Journal Collection Health | ISSN 2178-2091}

\begin{tabular}{|c|c|c|c|c|c|c|c|c|}
\hline \multirow[b]{2}{*}{ № } & \multirow[b]{2}{*}{ Base } & \multirow[b]{2}{*}{ Revista } & \multirow[b]{2}{*}{ Título } & \multirow[b]{2}{*}{ Autor (es) } & \multirow[b]{2}{*}{ Objetivo } & \multicolumn{2}{|c|}{ Metodologia } & \multirow[b]{2}{*}{ Ano } \\
\hline & & & & & & $\begin{array}{l}\text { Tipo de } \\
\text { estudo }\end{array}$ & Abordagem & \\
\hline 9 & PUBMED & $\begin{array}{l}\text { Journal of } \\
\text { Oncology } \\
\text { Practice }\end{array}$ & $\begin{array}{l}\text { A eficácia da acupressão para o controle } \\
\text { e gerenciamento de aguda e retardada } \\
\text { relacionada à náusea na quimioterapia: } \\
\text { um estudo controlado randomizado }\end{array}$ & $\begin{array}{l}\text { Molassiotis } \\
\text { A, et al. }\end{array}$ & $\begin{array}{c}\text { Avaliar um programa de massagem } \\
\text { integrativa de oncologia clínica para } \\
\text { pacientes submetidos a quimioterapia para } \\
\text { câncer de mama em um grande centro } \\
\text { médico acadêmico. }\end{array}$ & $\begin{array}{l}\text { Descritivo } \\
\text { exploratório }\end{array}$ & Quantitativa & 2017 \\
\hline 10 & SCIELO & Einstein & $\begin{array}{l}\text { Avaliação da aderência à diretriz de } \\
\text { cuidados para náuseas e vômitos } \\
\text { induzidos por quimioterapia }\end{array}$ & $\begin{array}{c}\text { França MS, } \\
\text { et al. }\end{array}$ & $\begin{array}{l}\text { Avaliar a adesão dos medicamentos } \\
\text { antieméticos prescritos em um centro } \\
\text { privado especializado em oncologia. }\end{array}$ & $\begin{array}{l}\text { Descritivo, } \\
\text { exploratório }\end{array}$ & Quantitativa & 2015 \\
\hline 11 & SCIELO & $\begin{array}{l}\text { Acta Paulista de } \\
\text { Enfermagem }\end{array}$ & $\begin{array}{l}\text { Efetividade de antieméticos no controle } \\
\text { da êmese induzida pela quimioterapia } \\
\text { antineoplásica, em domicílio }\end{array}$ & $\begin{array}{l}\text { Castro MC, } \\
\text { et al. }\end{array}$ & $\begin{array}{l}\text { Avaliar o impacto das drogas antiemeticas } \\
\text { atualmente utilizadas, sobre o controle da } \\
\text { emese em pacientes com cancer de mama } \\
\text { em tratamento quimioterapico de moderada } \\
\text { emetogenicidade. }\end{array}$ & $\begin{array}{l}\text { Observacional } \\
\text { longitudinal }\end{array}$ & Quantitativa & 2014 \\
\hline 12 & SCIELO & $\begin{array}{l}\text { Revista Gaúcha } \\
\text { de Enfermagem }\end{array}$ & $\begin{array}{l}\text { Ocorrência e manejo de náusea e } \\
\text { vômito no tratamento quimioterápico em } \\
\text { mulheres com câncer de mama }\end{array}$ & $\begin{array}{l}\text { Gozzo TO, } \\
\text { et al. }\end{array}$ & $\begin{array}{c}\text { Verificar a ocorrência de náusea e vômito } \\
\text { em mulheres com câncer de mama durante } \\
\text { a quimioterapia, e identificar o manejo } \\
\text { utilizado para o controle desses sinais e } \\
\text { sintomas. }\end{array}$ & Transversal & Quantitativa & 2014 \\
\hline 13 & SCIELO & $\begin{array}{l}\text { Revista Gaúcha } \\
\text { de Enfermagem }\end{array}$ & $\begin{array}{l}\text { Náuseas, vômitos e qualidade de vida } \\
\text { de mulheres com câncer de mama em } \\
\text { tratamento quimioterápico }\end{array}$ & $\begin{array}{l}\text { Gozzo TO, } \\
\text { et al. }\end{array}$ & $\begin{array}{l}\text { Avaliar a qualidade de vida de mulheres } \\
\text { com câncer de mama em tratamento } \\
\text { quimioterápico e mensurar a ocorrência de } \\
\text { episódios eméticos. }\end{array}$ & $\begin{array}{l}\text { Descritivo- } \\
\text { exploratório }\end{array}$ & Quantitativa & 2015 \\
\hline 14 & SCIELO & \begin{tabular}{|c|} 
Texto Contexto \\
Enfermagem
\end{tabular} & $\begin{array}{l}\text { Avaliação da qualidade de vida de } \\
\text { pacientes oncológicos em tratamento } \\
\text { quimioterápico adjuvante }\end{array}$ & $\begin{array}{c}\text { Machado } \\
\text { SM e } \\
\text { Sawada NO. }\end{array}$ & $\begin{array}{c}\text { Avaliar a qualidade de vida de pacientes } \\
\text { com câncer de mama e intestino, no início e } \\
\text { três meses após o tratamento } \\
\text { quimioterápico. }\end{array}$ & $\begin{array}{l}\text { Descritivo- } \\
\text { exploratório }\end{array}$ & Qualitativa & 2008 \\
\hline 15 & SCIELO & $\begin{array}{c}\text { Revista } \\
\text { Brasileira de } \\
\text { Enfermagem }\end{array}$ & $\begin{array}{c}\text { Utilização de experiências musicais } \\
\text { como terapia para sintomas de náusea e } \\
\text { vômito em quimioterapia }\end{array}$ & $\begin{array}{l}\text { Silva GJ, et } \\
\text { al. }\end{array}$ & $\begin{array}{l}\text { Aplicar experiências musicais e avaliar os } \\
\text { efeitos terapêuticos em episódio eméticos } \\
\text { causados pela quimioterapia antineoplásica. }\end{array}$ & $\begin{array}{l}\text { Descritivo, } \\
\text { transversal }\end{array}$ & Quantitativa & 2014 \\
\hline
\end{tabular}

Fonte: Gomes VC, et al., 2019. 


\section{Revista Eletrônica Acervo Saúde / Electronic Journal Collection Health | ISSN 2178-2091}

Quadro 2 - Caracterização dos estudos: Base, Título, Autor (es), Área de interesse, Formação e Titulação.

\begin{tabular}{|c|c|c|c|c|c|c|}
\hline № & Base & Título & Autor (es) & $\begin{array}{l}\text { Área de } \\
\text { interesse }\end{array}$ & Formação & Titulação \\
\hline 1 & BDENF & $\begin{array}{l}\text { O enfermeiro na prática educativa junto } \\
\text { aos familiares de clientes com câncer de } \\
\text { mama submetidas à quimioterapia }\end{array}$ & $\begin{array}{l}\text { Oliveira DFL } \\
\text { e Valente } \\
\text { GSC. }\end{array}$ & Enfermagem & Enfermeira (2) & $\begin{array}{l}\text { Doutora (1) } \\
\text { Graduação (1) }\end{array}$ \\
\hline 2 & BDENF & $\begin{array}{l}\text { Implementação de manuais educativos } \\
\text { na consulta de enfermagem: opinião dos } \\
\text { pacientes submetidos à quimioterapia } \\
\text { antineoplásica }\end{array}$ & $\begin{array}{l}\text { Cruz FOAM, } \\
\text { et al. }\end{array}$ & Enfermagem & Enfermeira (6) & $\begin{array}{l}\text { Doutora (1) } \\
\text { Doutoranda (1) } \\
\text { Mestre (3) } \\
\text { Mestranda (1) }\end{array}$ \\
\hline 3 & LILACS & $\begin{array}{l}\text { Mulheres com câncer de mama: ações } \\
\text { de autocuidado durante a quimioterapia }\end{array}$ & $\begin{array}{l}\text { Gonçalves } \\
\text { LLC, et al. }\end{array}$ & $\begin{array}{l}\text { Enfermagem } \\
\text { Estatística }\end{array}$ & $\begin{array}{c}\text { Enfermeira (4) } \\
\text { Estatístico (1) } \\
\text { Acadêmico de enfermagem (1) }\end{array}$ & $\begin{array}{c}\text { Mestre (3) } \\
\text { Metrando (1) } \\
\text { Especialista (1) } \\
\text { Graduando em Enfermagem } \\
\text { (1) }\end{array}$ \\
\hline 4 & MEDLINE & $\begin{array}{l}\text { Qualidade de vida e pessoas em uso de } \\
\text { antineoplásicos: estudo descritivo }\end{array}$ & Sá CU, et al. & Enfermagem & Enfermeira (5) & $\begin{array}{c}\text { Doutora (2) } \\
\text { Doutoranda (1) } \\
\text { Graduação (2) }\end{array}$ \\
\hline 5 & PUBMED & $\begin{array}{l}\text { Efeitos da massagem terapêutica na } \\
\text { qualidade de vida em pacientes com } \\
\text { câncer de mama durante o tratamento }\end{array}$ & $\begin{array}{l}\text { Sturgeon M, } \\
\text { et al. }\end{array}$ & $\begin{array}{l}\text { Medicina } \\
\text { Enfermagem } \\
\text { Psicologia }\end{array}$ & $\begin{array}{l}\text { Enfermeira (1) } \\
\text { Psicóloga (1) } \\
\text { Médico (2) }\end{array}$ & $\begin{array}{l}\text { Ph.d (2) } \\
\text { Doutor (2) }\end{array}$ \\
\hline 6 & PUBMED & $\begin{array}{c}\text { Efeito da terapia de ervas para náuseas } \\
\text { e vômitos induzidos por quimioterapia } \\
\text { em pacientes com câncer }\end{array}$ & $\begin{array}{l}\text { Montazeri } \\
\text { SA, et al. }\end{array}$ & $\begin{array}{l}\text { Farmácia } \\
\text { Enfermagem } \\
\text { Medicina }\end{array}$ & $\begin{array}{l}\text { Enfermeiros (2) } \\
\quad \text { Médico (1) } \\
\text { Farmacêutico (1) }\end{array}$ & $\begin{array}{l}\text { Ph.d (1) } \\
\text { Doutor (3) }\end{array}$ \\
\hline 7 & PUBMED & $\begin{array}{l}\text { Redução da anorexia, náusea e êmese } \\
\text { induzidas pela quimioterapia através de } \\
\text { uma intervenção estruturada de } \\
\text { enfermagem: um estudo multicêntrico } \\
\text { por grupo randomizado }\end{array}$ & Mao JJ, et al. & $\begin{array}{c}\text { Medicina } \\
\text { Enfermagem }\end{array}$ & $\begin{array}{c}\text { Médicos (2) } \\
\text { Enfermeiros (5) }\end{array}$ & $\begin{array}{l}\text { Doutor (4) } \\
\text { Mestre (3) }\end{array}$ \\
\hline
\end{tabular}

REAS/EJCH | Vol.Sup.n.53 | e3517 | DOI: https://doi.org/10.25248/reas.e3517.2020 Página 8 de 13 
Revista Eletrônica Acervo Saúde / Electronic Journal Collection Health | ISSN 2178-2091

\begin{tabular}{|c|c|c|c|c|c|c|}
\hline № & Base & Título & Autor (es) & $\begin{array}{c}\text { Área de } \\
\text { interesse }\end{array}$ & Formação & Titulação \\
\hline 8 & PUBMED & $\begin{array}{l}\text { Ingestão oral de gengibre para náuseas } \\
\text { e vômitos induzidos por quimioterapia } \\
\text { entre mulheres com câncer de mama }\end{array}$ & $\begin{array}{l}\text { Arslan M e } \\
\text { Ozdemir L. }\end{array}$ & Enfermagem & Enfermeira (2) & Ph.d (2) \\
\hline 9 & PUBMED & $\begin{array}{l}\text { A eficácia da acupressão para o controle } \\
\text { e gerenciamento de aguda e retardada } \\
\text { relacionada à náusea na quimioterapia: } \\
\text { um estudo controlado randomizado }\end{array}$ & $\begin{array}{c}\text { Molassiotis } \\
\text { A, et al. }\end{array}$ & $\begin{array}{c}\text { Enfermagem } \\
\text { Medicina }\end{array}$ & $\begin{array}{c}\text { Enfermeiros (3) } \\
\text { Médicos (3) } \\
\text { Acadêmicos de medicina (4) }\end{array}$ & $\begin{array}{c}\text { Ph.d (3) } \\
\text { Doutor (2) } \\
\text { Mestre (1) } \\
\text { Graduandos de medicina (4) }\end{array}$ \\
\hline 10 & SCIELO & $\begin{array}{l}\text { Efeitos da massagem terapêutica na } \\
\text { qualidade de vida em pacientes com } \\
\text { câncer de mama durante o tratamento }\end{array}$ & $\begin{array}{c}\text { França MS, } \\
\text { et al. }\end{array}$ & Medicina & Médico (8) & Graduação (8) \\
\hline 11 & SCIELO & $\begin{array}{c}\text { Efetividade de antieméticos no controle } \\
\text { da êmese induzida pela quimioterapia } \\
\text { antineoplásica, em domicílio }\end{array}$ & $\begin{array}{c}\text { Castro MC, } \\
\text { et al. }\end{array}$ & Enfermagem & Enfermeira (5) & $\begin{array}{c}\text { Doutor (2) } \\
\text { Mestre (2) } \\
\text { Especialista (1) }\end{array}$ \\
\hline 12 & SCIELO & $\begin{array}{c}\text { Ocorrência e manejo de náusea e } \\
\text { vômito no tratamento quimioterápico em } \\
\text { mulheres com câncer de mama }\end{array}$ & $\begin{array}{l}\text { Gozzo TO, et } \\
\text { al. }\end{array}$ & Enfermagem & Enfermeira (5) & $\begin{array}{c}\text { Doutora (1) } \\
\text { Mestre (2) } \\
\text { Mestranda (1) } \\
\text { Residente (1) } \\
\end{array}$ \\
\hline 13 & SCIELO & $\begin{array}{l}\text { Náuseas, vômitos e qualidade de vida } \\
\text { de mulheres com câncer de mama em } \\
\text { tratamento quimioterápico }\end{array}$ & $\begin{array}{l}\text { Gozzo TO, et } \\
\text { al. }\end{array}$ & Enfermagem & Enfermeira (4) & $\begin{array}{c}\text { Doutora (1) } \\
\text { Mestre (1) } \\
\text { Mestranda (2) }\end{array}$ \\
\hline 14 & SCIELO & $\begin{array}{c}\text { Avaliação da qualidade de vida de } \\
\text { pacientes oncológicos em tratamento } \\
\text { quimioterápico adjuvante }\end{array}$ & $\begin{array}{l}\text { Machado SM } \\
\text { e Sawada } \\
\text { NO. }\end{array}$ & Enfermagem & Enfermeira (2) & Doutora (2) \\
\hline 15 & SCIELO & $\begin{array}{c}\text { Utilização de experiências musicais } \\
\text { como terapia para sintomas de náusea e } \\
\text { vômito em quimioterapia }\end{array}$ & $\begin{array}{c}\text { Silva } \\
\text { GJ, et al. }\end{array}$ & Enfermagem & $\begin{array}{c}\text { Médico (1) } \\
\text { Enfermeiros (3) } \\
\text { Química (1) } \\
\text { Acadêmico de enfermagem (1) }\end{array}$ & $\begin{array}{l}\text { Doutora (2) } \\
\text { Graduação (3) } \\
\text { Graduando (1) }\end{array}$ \\
\hline
\end{tabular}

Fonte: Gomes VC, et al., 2019.

REAS/EJCH | Vol.Sup.n.53 | e3517 | DOI: https://doi.org/10.25248/reas.e3517.2020 Página 9 de 13 


\section{DISCUSSÃO}

O enfermeiro que atua em oncologia deve estar preparado para prestar uma assistência de qualidade, permitindo o desenvolvimento de estratégias convencionais ou não convencionais que auxilie de modo eficiente o bem-estar dos pacientes acometidos pela patologia (OLIVEIRA DFL e VALENTE GSC, 2014). Para isto, é necessário que o profissional tenha conhecimento suficiente sobre os protocolos quimioterápicos, o potencial emetogênico de cada droga, efeitos colaterais, o modo de administração, os fatores de risco individuais, o impacto desencadeado pelo tratamento e as características da Náusea e Vômito induzidos por Quimioterapia (NVIQ) (CASTRO MC, et al., 2014; CRUZ FOAM, et al., 2017).

Ao receber o diagnóstico, a paciente sofre impactos que desestruturam seu estado emocional, de certa forma interferindo no aprendizado cognitivo, desta maneira, dificultando o entendimento em relação ao seu tratamento. Esses fatores ocasionam aos pacientes e familiares momentos de grande angústia, incertezas, sofrimento, ansiedade, pois ocorre uma mudança repentina na vida de todos. A enfermagem exerce um papel de grande relevância na identificação e controle das náuseas e vômitos no paciente oncológico que está realizando quimioterapia, neste sentido, é fundamental que o enfermeiro especialista ofereça suporte através de estratégias de educação para conduzi-las ao conhecimento do processo saúde-doença, a fim de que tenham uma resposta positiva na adesão do tratamento (SÁ CU, et al., 2014).

Segundo o Instituto Nacional do Câncer (INCA), a mortalidade por câncer de mama no país é baixa quando comparada a países desenvolvidos como EUA, Canadá e Austrália, e melhor que alguns, como a França e Inglaterra, porém, é preciso avançar na prevenção, sobretudo na diminuição das desigualdades regionais e socioeconômicas. Neste contexto, observa-se que a mortalidade por câncer de mama está ligada principalmente ao acesso a diagnóstico imediato e na implantação do tratamento adequado, uma vez que é uma doença com grande potencial de cura caso diagnosticada e tratada precocemente (INCA, 2019).

O enfrentamento do câncer é um dos principais desafios para pacientes e familiares, ultrapassando as barreiras familiares em muitos casos, pois vizinhos, colegas de trabalho, amigos e pessoas próximas da paciente vivenciam tanto o impacto com o diagnóstico quanto os sentimentos de impotência e incerteza mediante o tratamento e consequências futuras. Dentre as estratégias de enfrentamento, temos a prática religiosa, que vem sendo exercida há gerações. É preciso força espiritual para manter a fé durante o tratamento, desde a confirmação do diagnóstico e aceitação do quadro clínico, o paciente recorre a fé e as orações para um ser superior e atribui a ele cada vitória conseguida nas etapas da doença, além da motivação para vencer cada obstáculo (FERREIRA NML, et al., 2010).

Em muitos casos, as pessoas por estarem acometidas por alguma doença, tendem a ficar mais sensíveis, se apoderando daquilo que acreditam trazer benefícios visando a reabilitação, neste sentido, em um estudo realizado com mulheres diagnosticadas com câncer no interior de São Paulo, notou-se que a religiosidade e a fé em Deus mostrou-se uma eficiente medida de conforto e segurança no enfrentamento do câncer (PANOBIANCO MS, et al., 2012).

Uma das etapas mais delicadas do tratamento de câncer é a quimioterapia, no estudo de Gonçalves LLC, et al. (2009), referente ao conhecimento das mulheres com câncer de mama sobre a quimioterapia, foi identificado que $81,04 \%$ das pacientes alegavam não possuir conhecimento algum sobre os efeitos colaterais da quimioterapia, além disto, a ocorrência de náuseas e vômitos foi o efeito colateral mais citado $(74,13 \%)$.

As náuseas e vômitos induzidos por antineoplásicos impactam negativamente na qualidade de vida de pessoas em tratamento quimioterápico. Durante esse período surgem sintomas indesejados como fadiga e insônia, que são agravados com a ocorrência de náusea e vômito, acarretando limitações na vida do indivíduo, dentre elas: físicas, como realizar atividades domésticas ou laborais, lazer, como praticar atividades relaxantes e esportivas, realizar o autocuidado e ser incapaz de tomar as medicações prescritas (SÁ CU, et al., 2014).

A partir deste dado científico, nota-se a importância da ação de enfermagem frente à prática da educação em saúde, pois o profissional da categoria é o principal facilitador de informações fidedignas e contínuas que podem influenciar diretamente na conduta das pacientes durante o tratamento, quando relacionado à prevenção e controle de NVIQ (GOZZO TO, et al., 2013). 
Para auxiliar na divulgação de informações, durante as consultas de enfermagem, algumas medidas alternativas de práticas educacionais podem ser implantadas, através de manuais educativos, que contenham informações autoexplicativas sobre: alimentação, hidratação, risco de infecção, efeitos colaterais, prevenção e controle de náuseas agudas e tardias, com uma linguagem simplificada que possa esclarecer dúvidas que os pacientes apresentam, pois na maioria das vezes não são esclarecidas por motivo de esquecimento ou timidez (CRUZ FOAM, et al., 2017).

De acordo com Almeida RGL, et al. (2015), durante a prestação de cuidados aos pacientes oncológicos que estão realizando quimioterapia, é fundamental preservar a qualidade de vida através do manejo correto das náuseas e vômitos desde o início, visto que o controle ineficaz desse manejo poderá implicar no aparecimento desses agravos de forma tardia, ocasionando impactos negativos na qualidade de vida, podendo ainda gerar aumento dos custos nos serviços de saúde, com a utilização de outras medicações, realização de consultas médicas não agendadas e internações desnecessárias.

Alguns estudos destacam que existe um número significativo de pacientes que não aderem o uso de antiemético, quando indicados para serem utilizados em domicílio (antes do ciclo quimioterápico ou depois), isto ocorre devido à falta de conhecimento sobre a importância que os mesmos têm em reduzir os sintomas indesejados, além de fazerem uso da medicação de forma irregular (GOZZO TO, et al., 2014; FRANÇA MS, et al., 2015).

Diversos antieméticos são aplicados na prevenção e tratamento de náusea e êmese induzida pela terapia antineoplásica, os mais utilizados no Brasil são: Ondasetrona e Dexametasona. Estas drogas devem ser utilizadas corretamente, obedecendo ao padrão do horário certo e dose certa, para que os episódios de náuseas e vômitos sejam reduzidos consideravelmente (BECKER J e NARDIN JM, 2011).

O uso incorreto dos medicamentos traz prejuízos à saúde das pacientes, resultando no agravo da doença. Os efeitos colaterais podem ser tão estressantes a ponto de causar adiamento ou suspensão do ciclo quimioterápico, e consequentemente, ocasionar atraso no tratamento, que por sua vez, destrói as células neoplásicas. Neste contexto, Castro MC, et al. (2014), aborda que durante a realização da assistência de enfermagem oncológica, é possível associar o manejo farmacológico da medicina com condutas não farmacológicas (acupuntura, relaxamento, homeopatia, aromoterapia e outros), que de certa forma irão auxiliar na melhora dos sintomas induzidos pela TA.

Nos estudos experimentais de Montazeri AS, et al. (2013) e Arslan M, Ozdemir L (2015), o uso do gengibre apresentou um resultado significativo no controle de náusea e vômito, sendo utilizado o placebo e gengibre em cápsula, ingeridos por via oral. De acordo com Montazeri AS, et al. (2013), o gengibre foi 18,4\% mais efetivo do que o placebo. Já Arslan M, Ozdemir L (2015), aplicou em seu estudo $500 \mathrm{mg}$ de gengibre em pó duas vezes ao dia, por três dias consecutivos em mulheres com câncer de mama, a eficácia do estudo experimental demonstrou segurança durante o uso do fitoterápico, sem mostrar nenhum efeito colateral.

Para Molassiotis A, et al. (2014), a utilização da acupuntura tem um grande potencial a ser explorado para o controle da Náusea e Vômito, portanto, este tipo de medicina alternativa tem demostrado resultados positivos ao ser realizado em pacientes oncológicos sem que lhes causem danos ou prejuízos, pois trata-se da medicina baseada em evidências e agrega valor ao conjunto de opções benéficas que atenda a mulher de forma holística.

Em um estudo desenvolvido num centro médico acadêmico da Pensilvânia (EUA), foi proposto um programa de massagem oncológica clínica nos ambulatórios de quimioterapia, para as pacientes com neoplasia mamária. O estudo demostrou que a massagem terapêutica pode ser integrada de forma segura e eficaz no controle dos eventos adversos indesejados e que o uso concomitante da terapia antineoplásica com a massagem contribui na redução não apenas de NVIQ, mas também no controle da fadiga, ansiedade e qualidade do sono, refletindo na melhora da qualidade de vida (MAO JJ, et al., 2017). Achados corroborados pelo estudo de Sturgeon M, et al. (2009), que apontou características semelhantes, sendo desenvolvido em Wichita, no Estado do Kansas (EUA).

O uso terapêutico da música mostra-se uma estratégia complementar importante, que pode trazer contribuições significativas, como o controle da dor, ansiedade, promoção do relaxamento, diminuindo assim a ocorrência dos episódios eméticos. Porém, a falta de preparo dos profissionais de saúde para lidar com tais 
terapias e métodos alternativos, de certa forma acabam deixando de utilizar essas práticas, fazendo com que os efeitos positivos sejam eles psicológicos ou físicos, não possam ser oferecidos aos pacientes. Também é importante ressaltar que os benefícios se trata de estratégias de baixo custo, além da possibilidade de ofertar uma prática não farmacológica e não invasiva (SILVA GJ, et al., 2014).

Em virtude de diversas opções de técnicas não farmacológicas, a enfermagem tem a possibilidade de agregar ao manejo farmacológico um conjunto de terapias complementares, a fim de aumentar a melhora na qualidade de vida das mulheres que passam por momentos de fragilidades durante o seu tratamento. Neste panorama, a atuação do enfermeiro na esfera da qualidade de assistência, possibilita menor sobrecarga imposta a cliente, acrescentando a diversificação de técnicas e aplicação de métodos médicos não convencionais de medicina alternativa, que busca não especificamente a cura, mas também o bem-estar, auxiliando-a no enfrentamento do diagnóstico e tratamento da doença (MACHADO SM e SAWADA NO, 2008).

\section{CONSIDERAÇÕES FINAIS}

A partir deste estudo, foi possível perceber que a atuação do enfermeiro frente ao manejo de náuseas e vômitos em mulheres com câncer de mama que realizam terapia antineoplásica, se confunde com as ações de cuidados gerais de outros profissionais. Neste sentido, o estudo apontou que as principais condutas no manejo dessas mulheres estão baseadas na implementação de atividades educativas que promovam o conhecimento adequado da paciente em relação ao tratamento e seus possíveis efeitos colaterais, frisando a importância das medidas farmacológicas (antieméticos) para a prevenção e controle de NVIQ, associados a implementação de terapias não farmacológicas: massagens terapêuticas, acupuntura e musicoterapia.

\section{REFERÊNCIAS}

1. ALMEIDA RGL, et al. O manejo da êmese em uma unidade oncológica: a necessidade da intervenção farmacêutica em tempo real. Rev Bras Cancerol. [periódico na Internet]. 2015.

2. ARSLAN M e OZDEMIR L. Oral Intake of Ginger for Chemotherapy-Induced Nausea and Vomiting Among Women with Breast Cancer. Clinical Journal of Oncology Nursing. 2015; 19(5): 92-97.

3. BECKER J e NARDIN JM. Utilização de antieméticos no tratamento antineoplásico de pacientes oncológico. R. Bras. Farm. Hosp. Serv. Saúde São Paulo. 2011; 2(3): 18-22.

4. BRASIL. Ministério da Saúde. Instituto Nacional do Câncer. ABC do câncer: abordagens básicas para o controle do câncer, 4. Ed. Rio de Janeiro 2018.

5. BRASIL. Ministério da Saúde. Secretaria de Atenção à Saúde. Núcleo Técnico da Política Nacional de Humanização. HumanizaSUS: documento base para gestores e trabalhadores do SUS. 4. ed. Brasília: Ministério da Saúde, 2008.

6. CASTRO MC, et al. Effectiveness of antiemetics in control of antineoplastic chemotherapy-induced emesis at home. Acta Paulista de Enfermagem. 2014; 27(5): 412-418.

7. CONCEIÇÃO LL, et al. O cotidiano de mulheres mastectomizadas: do diagnóstico à quimioterapia. Revista de Enfermagem UERJ. Rio de Janeiro. 2008; 1(16): 26-31.

8. CRUZ FOAM, et al. Implementação de manuais educativos na consulta de enfermagem: opinião dos pacientes submetidos à quimioterapia antineoplásica. Revista de Enfermagem UFPE Online, Recife. 2017; 5(11): $1757-1762$.

9. FERNANDES DT, et al. Mulheres mastectomizadas em vigência de quimioterapia adjuvante: assistência do enfermeiro. Biológicas \& Saúde. 2018; 8(26): 19-29.

10. FERREIRA NML, et al. Câncer e família: compreendendo os significados simbólicos. Ciênc Cuid Saúde. 2010.

11. FRANÇA MS, et al. Assessment of adherence to the guidelines for the management of nausea and vomiting induced by chemotherapy. Einstein (são Paulo). 2015; 13(2): 221-225.

12. FRAZÃO A e SKABA MMF. Mulheres com Câncer de Mama: as Expressões da Questão Social durante o Tratamento de Quimioterapia Neoadjuvante. Revista Brasileira de Cancerologia. Rio de Janeiro. 2013; 3(59): 427-435.

13. GONÇALVES LLC, et al. Mulheres com câncer de mama: ações de autocuidado durante a quimioterapia. Revista de Enfermagem Uerj. Rio de Janeiro. 2009; 4(17): 575-580.

14. GOZZO TO, et al. Náuseas, vômitos e qualidade de vida de mulheres com câncer de mama em tratamento quimioterápico. Revista Gaúcha de Enfermagem. 2013; 34(3): 110-116.

15. GOZZO TO, et al. Incidence and management of chemotherapy-induced nausea and vomiting in women with breast cancer. Revista Gaúcha de Enfermagem. 2014; 35(3): 117-123.

16. INCA - INSTITUTO NACIONAL DO CÂNCER. Câncer de mama. Rio de Janeiro, 2018.

17. INCA - INSTITUTO NACIONAL DO CÂNCER. Estimativas 2018. Incidência de câncer no Brasil. Rio de Janeiro, 2017.

18. INSTITUTO NACIONAL DE CÂNCER (Brasil). Estimativa 2020. Incidência do Câncer no Brasil. Rio de Janeiro: INCA, 2019.

19. MAO JJ, et al. Integrating Oncology Massage Into Chemoinfusion Suites: A Program Evaluation. Journal of Oncology Practice. 2017; 13(3): 207-216. 
20. MACHADO SM e SAWADA NO. Avaliação da qualidade de vida de pacientes oncológicos em tratamento quimioterápico adjuvante. Texto \& Contexto - Enfermagem. 2008; 17(4): 750-757.

21. MIGOWSKI A, et al. Diretrizes para detecção precoce do câncer de mama no Brasil. II - Novas recomendações nacionais, principais evidências e controvérsias. Cadernos de Saúde Pública. 2018; 34(6): 3-16.

22. MOLASSIOTIS A, et al. The Effectiveness of Acupressure for the Control and Management of Chemotherapy-Related Acute and Delayed Nausea: A Randomized Controlled Trial. Journal of Pain and Symptom Management. 2014; 47(1): $12-25$.

23. MONTAZERI AS, et al. Effect of Herbal Therapy to Intensity Chemotherapy-Induced Nausea and Vomiting in Cancer Patients. Iranian Red Crescent Medical Journal. 2013; 15(2): 101-6.

24. OLIVEIRA DFL e VALENTE GSC. O enfermeiro na prática educativa junto aos familiares de clientes com câncer de mama submetidas à quimioterapia. Revista de Enfermagem UFPE Online, Recife. 2014; 3(8): 4071-4079.

25. OPAS/WHO - ORGANIZAÇÃO MUNDIAL DA SAÚDE/ORGANIZAÇÃO PAN-AMERICANA DA SAÚDE. Folha informativa - Câncer. Brasil, 2018.

26. PANOBIANCO MS, et al. Mulheres com diagnóstico avançado do câncer do colo do útero: enfrentando a doença e o tratamento. Rev bras cancerol. 2012.

27. SÁ CU, et al. Quality of life and people using antineoplastic agents: a descriptive study. Online Brazilian Journal of Nursing. 2014; 13(4): 579-589.

28. SILVA GJ, et al. Utilização de experiências musicais como terapia para sintomas de náusea e vômito em quimioterapia. Revista Brasileira de Enfermagem. 2014; 67(4): 630-636.

29. SOUZA MT, et al. Revisão integrativa: o que é e como fazer. Einstein, Sao Paulo. 2010; 4(8): $102-106$.

30. SOARES CB, et al. Revisão integrativa: conceitos e métodos utilizados na enfermagem. Revista Escola de Enfermagem da USP, São Paulo. 2014; 2(48): 335-345.

31. STURGEON M, et al. Effects of Therapeutic Massage on the Quality of Life Among Patients with Breast Cancer During Treatment. The Journal of Alternative and Complementary Medicine. 2009; 15(4): 373-380. 